Pacific Journal of Mathematic 


\title{
HIGHER DIMENSIONAL CYCLIC ELEMENTS
}

\author{
JOHN GARY
}

Introduction. Whyburn, in 1934, introduced the higher dimensional cyclic elements [5]. He gave an analysis of the structure of the homology groups of a space in terms of its cyclic elements. His results were for finite dimensional spaces, and he used the integers modulo two as the coefficient group. Puckett generalized some of Whyburn's results to compact metric spaces [3]. Simon has shown that if $E$ is a closed subset of a compact space $M$, which contains all the $(r-1)$-dimensional cyclic elements of $M$, then $H^{r}(E) \approx H^{r}(M)[4]$. He also obtained a direct sum decomposition of $H^{r}(M)$ using the cyclic elements of $M$. We will extend some of these results.

The properties of zero-dimensional cyclic elements in locally connected spaces, and the relation of these cyclic elements to monotone mappings, is basic in the applications of zero-dimensional cyclic element theory. We shall give some counter-examples concerning the generalization of these properties to higher dimensional cyclic elements.

1. Preliminaries. Throughout this paper $M$ will always denote a compact Hausdorff space. We shall use the augmented Cech homology and cohomology with a field as coefficient group. Results stated in terms of cohomology may be given a dual expression in terms of homology by means of the dot product duality for the Cech theory.

Definition 1.1. A $T_{r}$ set in $M$ is a closed subset $T$ of $M$ such that $H^{r}(K)=0$, for all closed subsets $K$ of $T$.

Definition 1.2. An $E_{r}$ set in $M$ is a non-degenerate subset of $M$ which is maximal with respect to the property that it can not be disconnected by a $T_{r}$ set of $M$.

The proofs of Lemmas 1.3 through 1.9 can be found in the papers by Whyburn [5] and Simon [4]. The proofs given by Whyburn are for subsets of Euclidean space, but they can be carried over to our case without difficulty.

Lemma 1.3. Let $K$ be a subset of $M$ which can not be disconnected by a $T_{r}$ set. If $M=M_{1} \cup M_{2}, T_{r}$-separated (by this we mean $M_{1}$ and $M_{2}$ are proper closed subsets and $M_{1} \cap M_{2}$ is a $T_{r}$ set), then $K \subset M_{1}$ (or, $\left.K \subset M_{2}\right)$.

Received December 24, 1958. The results of this paper are contained in the authors doctoral dissertation, University of Michigan, 1956. The author wishes to thank Professor R. L. Wilder for his advice and encouragement. 
Lemma 1.4. If $K$ is an $E_{r}$ set, then $K$ is closed and connected.

Lemma 1.5. If $K_{1}$ and $K_{2}$ are both $E_{r}$ sets and $K_{1} \neq K_{2}$, then $K_{1} \cap K_{2}$ is a $T_{r}$ set. Any $T_{r}$ set is also a $T_{r+1}$ set.

Lemma 1.6. If $K$ is a non-degenerate subset of $M$, which can not be disconnected by a $T_{r}$ set, then $K$ is contained in a unique $E_{r}$ set in $M$.

DEFINITION 1.7. If $\gamma^{r} \in H^{r}(M)$ and $D$ is a minimal, closed subset of $M$ such that $i^{*}\left(\gamma^{r}\right) \neq 0$ (where $i^{*}: H^{r}(M) \rightarrow H^{r}(D)$ is the inclusion map), then $D$ is called a floor for $\gamma^{r}$.

Lemma 1.8. If $\gamma^{r} \in H^{r}(M)$ and $\gamma^{r} \neq 0$, then there exists a floor for $\gamma^{r}$.

LemMa 1.9. If $D$ is a floor for $\gamma^{r}$, then $D$ can not be disconnected by a $T_{r-1}$ set.

Lemma 1.10. If $\left\{E^{1}, \cdots, E^{n}\right\}$ is a finite collection of $E_{r-1}$ sets in $M$, with $M \neq \bigcup_{i=1}^{n} E^{i}$, then there exist proper, closed subsets, $M_{1}$ and $M_{2}$, of $M$ such that (1) $M=M_{1} \cup M_{2}$, (2) $M_{1} \cap M_{2}$ is the union of a finite number of $T_{r-1}$ sets (therefore, $M_{1} \cap M_{2}$ is a $T_{r}$ set), (3) $M_{1} \supset \bigcup_{i=1}^{n} E^{i}$.

Proof. The proof will be by induction on $n$. The case $n=1$ follows from Lemma 1.3 .

Assume the lemma is true up to $n-1$. Since $M$ is not an $E_{r-1}$ set, we have $M=M_{1} \cup M_{2}, T_{r-1}$-separated. Let $E=\mathrm{U}_{i=1}^{n} E^{i}$. If $(M-E) \cap\left(M-\left(M_{1} \cap M_{2}\right)\right)=\phi$, then the desired $T_{r-1}$-separation of $M$ could be obtained by using the boundary of an open set in $M_{1} \cap M_{2}$. Therefore, we can assume $(M-E) \cap\left(M-M_{1}\right) \neq \phi$. By Lemma 1.3, we can assume $\bigcup_{i=1}^{s} E^{i} \subset M_{2}$ and $\bigcup_{i=s+1}^{n} E^{i} \subset M_{1}$, where $1 \leq s<n$. We must have $E^{i} \subset\left(\overline{M-M_{1}}\right)$, for $1 \leqq i \leqq s$. Otherwise, we could separate $E^{i}$ by the $T_{r-1}$ set $\left(\overline{M-M_{1}}\right) \cap\left(M_{1} \cap M_{2}\right)$. Since $(M-E) \cap\left(M-M_{1}\right) \neq \phi$, $\left(\overline{M-M_{1}}\right) \neq \bigcup_{i=1}^{s} E^{i}$. Thus, by the induction assumption, $\left(\overline{M-M_{1}}\right)=$ $M_{4} \cup M_{5}$, where $\bigcup_{i=1}^{s} E^{i}$ is contained in $M_{4}$ and $M_{4} \cap M_{5}$ is the union of a finite number of $T_{r-1}$ sets. If we let $\tilde{M}_{1}=M_{1} \cup M_{4}$ and $\tilde{M}_{2}=M_{5}$, then

(1) $M=\tilde{M}_{1} \cup \tilde{M}_{2}$

(2) $\tilde{M}_{1} \cap \tilde{M}_{2}$ is the union of a finite number of $T_{r-1}$ sets,

(3) $\bigcup_{i=1}^{n} E^{i} \subset \tilde{M}_{1}$,

(4) $\widetilde{M}_{1}$ and $\tilde{M}_{2}$ are proper closed subsets of $M$. 


\section{Cyclic elements and the structure of $M$.}

Definition 2.1. A closed subset $A$ of $M$ is called a $L_{r}$ set if every $E_{r-1}$ set, whose intersection with $A$ is not a $T_{r}$ set, is contained in $A$. The proofs of the following theorems are given below.

Theorem 2.2. If $A$ is a $L_{r}$ set, then $i^{*}: H^{r}(M) \rightarrow H^{r}(A)$ is onto. Thus, by duality, $i_{*}: H_{r}(A) \rightarrow H_{r}(M)$ is one-to-one.

THEOREM 2.3. Let $A$ be a closed subet with the following property: if $E$ is an $E_{r-1}$ set and $H^{r}(E) \neq 0$, then $E$ is contained in $A$. Then the $\operatorname{map} i^{*}: H^{r}(M) \rightarrow H^{r}(A)$ is one-to-one and, by duality, $i_{*}: H_{r}(A) \rightarrow$ $H_{r}(M)$ is onto.

THEOREM 2.4. Suppose there are only a finite number, say $\left\{E^{1}, \cdots, E^{n}\right\}$, of $E_{r-1}$ sets such that $H^{r}\left(E^{i}\right) \neq 0$. Let $A=\bigcup_{i=1}^{n} E^{i}$. Then the mappings $i^{*}: H^{r}(M) \rightarrow H^{r}(A)$ and $i_{*}: H_{r}(A) \rightarrow H_{r}(A)$ are isomorphisms.

REMARK. Theorem 2.4 can not be generalized to an infinite number of $E_{r-1}$ sets, as the following example shows. In Euclidean space let $M=D \cup\left[\bigcup_{i=1}^{\infty} C_{i}\right]$, where $D=\left\{(x, y, z) \mid z=0, x^{2}+y^{2} \leqq 1\right\}$ and $C_{i}=$ $\left\{(x, y, z) \mid z=1 / i, x^{2}+y^{2}=1\right\}$. We do not have $H_{1}\left(\bigcup_{i=1}^{\infty} C_{i}\right) \approx H_{1}(M)$, under the inclusion mapping.

THEOREM 2.5. Let $\gamma^{r} \in H^{r}(M)$ and suppose $U$ is an open set, such that if $D$ is a floor for $\gamma^{r}$, then $D$ is contained in $U$ (see Definition 1.7). Then there exists a $\gamma_{u}^{r} \in H^{r}(M, M-U)$ such that $\gamma^{r}=j^{*}\left(\gamma_{u}^{r}\right)$, where $j^{*}: H^{r}(M, M-U) \rightarrow H^{r}(M)$.

Theorem 2.6. Assume $E$ is an $E_{r-1}$ set in $M$ and $N$ is a closed subset of $M$, where $N \cap E=\phi$. Then the composite mapping $j_{*} i_{*}: H_{r}(E) \rightarrow$ $H_{r}(M, N)$ is one-to-one. Here, $i_{*}: H_{r}(E) \rightarrow H_{r}(M)$ and $j_{*}: H_{r}(M) \rightarrow$ $H_{r}(M, N)$ are the natural mappings.

Lemma 2.7. Let $(M, A)$ be a compact pair with $\gamma^{r} \in H^{r}(A)$. If $\delta^{*}\left(\gamma^{r}\right) \neq 0$, where $\delta^{*}: H^{r}(A) \rightarrow H^{r+1}(M, A)$, then there is a minimal closed set $B$ such that $B \subset A$, and $\delta_{B}^{*}\left(\gamma_{B}^{r}\right) \neq 0$. Here, $\delta_{B}^{*}: H^{r}(B) \rightarrow$ $H^{r+1}(M, B)$ and $\gamma_{B}^{r}=i^{*}\left(\gamma^{r}\right)$, where $i^{*}: H^{r}(A) \rightarrow H^{r}(B)$.

Lemma 2.8. Let $B$ be a minimal set defined in Lemma 2.7. There exists a minimal closed set $N$ such that $\delta^{*}\left(\gamma_{B}^{r}\right) \neq 0$, where $\delta^{*}: H^{r}(B) \rightarrow$ $H^{r+1}(N, B)$. 
Proof. The proof of these lemmas is obtained from the continuity of the Cech theory and Zorn's lemma.

Lemma 2.9. The set $N$, in Lemma 2.8, can not be disconnected by a $T_{r-1}$ set.

Proof. Suppose $N=N_{1} \cup N_{2}$, where $N_{1} \cap N_{2}$ is a $T_{r-1}$ set. We will show this to be impossible, unless $N=N_{1}$. Let $B$ be as defined in Lemma 2.8, and define $B_{i}=N_{i} \cap B(i=1,2)$. We will show that the mapping induced by inclusion

$$
K^{*}: H^{r+1}(N, B) \rightarrow H^{r+1}\left(N_{1}, B_{1}\right) \oplus H^{r+1}\left(N_{2}, B_{2}\right)
$$

is an isomorphism. We use the relative Mayer-Vietoris sequence given below; note that $T=N_{1} \cap N_{2}$ is a $T_{r-1}$ set [2].

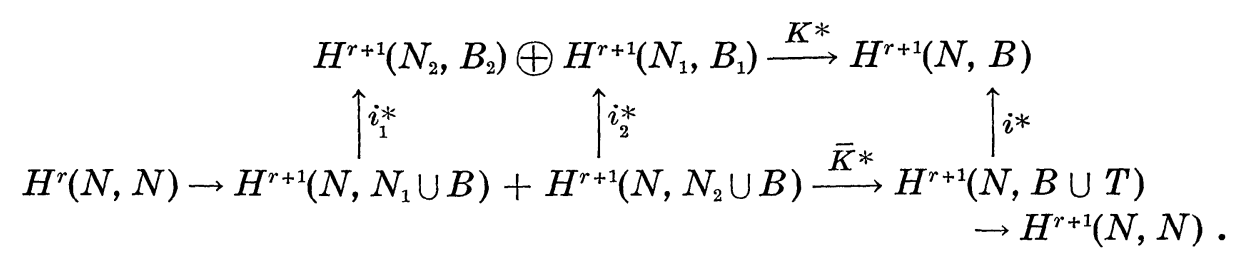

The mappings $i_{1}^{*}$ and $i_{2}^{*}$ are isomorphisms by excision, the map $\bar{K}^{*}$ by exactness. Using the three exact sequences given below we see that $i^{*}$ is an isomorphism.

$$
\begin{gathered}
H^{s-1}(B \cap T) \rightarrow H^{s}(B \cup T) \rightarrow H^{s}(B) \oplus H^{s}(T) \rightarrow H^{s}(B \cap T) \\
H^{s}(B \cup T) \rightarrow H^{s}(B) \rightarrow H^{s+1}(B \cup T, B) \rightarrow H^{s+1}(B \cup T) \rightarrow H^{s+1}(B) \\
H^{r}(B \cup T, B) \rightarrow H^{r+1}(N, B \cup T) \rightarrow H^{r+1}(N, B) \rightarrow H^{r+1}(B \cup T, B) .
\end{gathered}
$$

The first is a Mayer-Vietoris sequence, the second is a sequence for a pair, the third is a sequence for a triple. Thus $K^{*}$ is an isomorphism. In the diagram below, since $\delta_{N}^{*}\left(\gamma_{B}^{r}\right) \neq 0$, we may assume $\delta_{N_{1}}^{*} \phi_{1}^{*}\left(\gamma_{B}^{r}\right) \neq 0$.

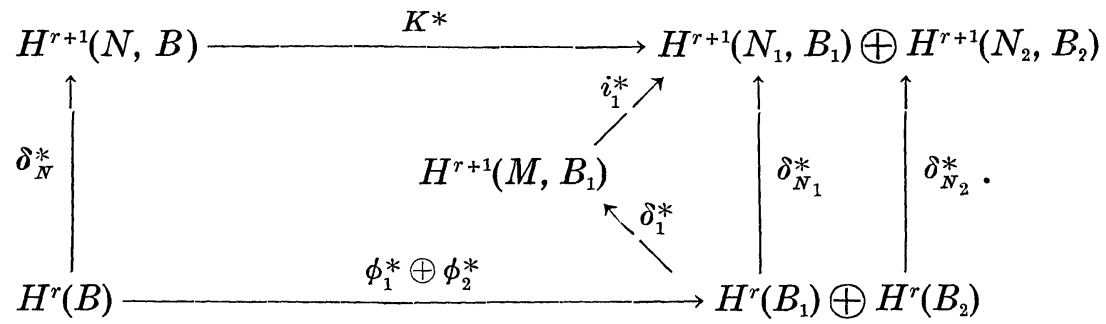

We now have $\delta_{1}^{*} \phi_{1}^{*}\left(\gamma_{B}^{r}\right) \neq 0$, since $i_{1}^{*} \delta_{1}^{*} \phi_{1}^{*}\left(\gamma_{B}^{r}\right)=\delta_{N_{1}}^{*} \phi_{1}^{*}\left(\gamma_{B}^{r}\right) \neq 0$. This implies $B_{1}=B$, by the definition of $B$. Therefore, $\phi_{1}^{*}\left(\gamma_{B}^{r}\right)=\gamma_{B}^{r}$ and $\delta_{N_{1}}^{*} \phi_{1}^{*}\left(\gamma_{B}^{r}\right)=\delta_{N_{1}}^{*}\left(\gamma_{B}^{r}\right) \neq 0$. Since $N$ is minimal, we must have $N_{1}=N$. Thus, $N$ can not be disconnected by a $T_{r-1}$ set. 
Proof of Theorem 2.2. We will show $\delta^{*}\left(\gamma^{r}\right)=0$, for all $\gamma^{r} \in H^{r}(A)$, where $\delta^{*}: H^{r}(A) \rightarrow H^{r}(M, A)$. Suppose not; then choose $N$ and $B$ according to Lemma 2.8. Then there exists an $E_{r-1}$ set containing $N$, by Lemma 1.6. Let $E$ denote this $E_{r-1}$ set. Since $E$ contains $N$, we have $E \cap A \supset B$. Since $H^{r}(B) \neq 0, B$ is not a $T_{r}$ set. Therefore $E \subset A$, because $A$ is an $L_{r}$ set. This implies that $N$ is contained in $A$. But this is impossible, as the diagram below shows. By the definition of the pair $(N, B), \delta^{*} i^{*}\left(\gamma^{r}\right) \neq 0$.

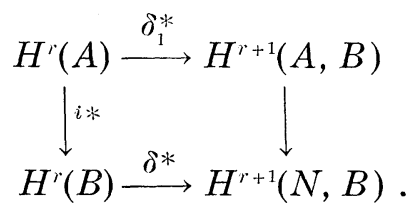

Proof of Theorem 2.3. Consider the exact sequence:

$$
H^{r}(M, A) \stackrel{j^{*}}{\longrightarrow} H^{r}(M) \stackrel{i^{*}}{\longrightarrow} H^{r}(A) .
$$

Suppose $j^{*}\left(\gamma^{r}\right) \neq 0$, where $\gamma^{r} \in H^{r}(M, A)$. By Lemmas 1.9 and 1.6 there is an $E_{r-1}$ set which contains a floor for $j^{*}\left(\gamma^{r}\right)$. Let $E$ be this $E_{r-1}$ set. Since $E$ contains a floor for $j^{*}\left(\gamma^{r}\right), H^{r}(E) \neq 0$. Therefore, $E \subset A$; which implies $i^{*} j^{*}\left(\gamma^{*}\right) \neq 0$, since $E$ contains a floor for $j^{*}\left(\gamma^{r}\right)$. Therefore $j^{*}$ is a trivial map and $i^{*}$ is one-to-one.

Proof of Theorem 2.4. By Theorem 2.3, $i^{*}: H_{r}(A) \rightarrow H_{r}(M)$ is onto. If $i_{*}\left(Z_{r}\right)=0$, for some $Z_{r} \in H_{r}(A)$; then there is a minimal set $K$ such that

(1) $K \supset A$, and

(2) $i_{*}^{K}\left(Z_{r}\right)=0$, where $i_{*}^{K}: H_{r}(A) \rightarrow H_{r}(K)$ [2]. If $K \neq A$; then, by Lemma 1.10 , we have $K=K_{1} \cup K_{2}, T_{r}$-separated. The Mayer-Vietoris sequence below implies $i_{*^{K}}^{K}\left(Z_{r}\right)=0$, where $i_{*^{1}}^{K}: H_{r}(A) \rightarrow H_{r}\left(K_{1}\right)$.

$$
H_{r}\left(K_{1} \cap K_{2}\right) \rightarrow H_{r}\left(K_{1}\right) \oplus H_{r}\left(K_{2}\right) \rightarrow H_{r}(K) .
$$

Therefore, $K=A$ and $Z_{r}=0$, or $i_{*}$ is one-to-one.

Proof of Theorem 2.5. Consider the exact sequence,

$$
H^{r}(M, M-U) \stackrel{j^{*}}{\longrightarrow} H^{r}(M) \stackrel{i^{*}}{\longrightarrow} H^{r}(M-U) .
$$

We will show $i^{*}\left(\gamma^{r}\right)=0$, where $\gamma^{r}$ is the element of $H^{r}(M)$ given in the theorem. Suppose $i^{*}\left(\gamma^{r}\right) \neq 0$; then, by Lemma 1.8, there exists a floor for $i^{*}\left(\gamma^{r}\right)$ contained in $M-U$. If $D$ is this floor, then $D$ is a floor for $\gamma^{r}$, since $i_{D}^{*}=i_{D U}^{*} i^{*}$. Here, $i_{D}^{*}: H^{r}(M) \rightarrow H^{r}(D)$ and $i_{D U}^{*}: H^{r}(M-U) \rightarrow$ $H^{r}(D)$ are inclusion mappings. Therefore, by the definition of $U, D$ is contained in $U$. This is impossible, hence $i^{*}\left(\gamma^{r}\right)=0$. 
Proof of Theorem 2.6. Let $\phi_{*}=j_{*} i_{*}$, and suppose $\phi_{*}\left(Z_{r}\right)=0$, for some $Z_{r} \in H_{r}(E)$. Then there exists a minimal closed set $K$ in $M$ such that $K \supset E$ and $\phi_{*}^{K}\left(Z_{r}\right)=0$, where $\phi_{*}^{K}: H_{r}(E) \rightarrow H_{r}(K, K \cap N)$ is analogous to $\phi_{*}$ defined above. This follows from Zorn's lemma and continuity. We will assume $K \neq E$. Since $E$ is an $E_{r-1}$ set, we can write $K=K_{1} \cup K_{2}, T_{r-1}$-separated. Also, we can assume $E \subset K_{1}$. Consider the following commutative diagram:

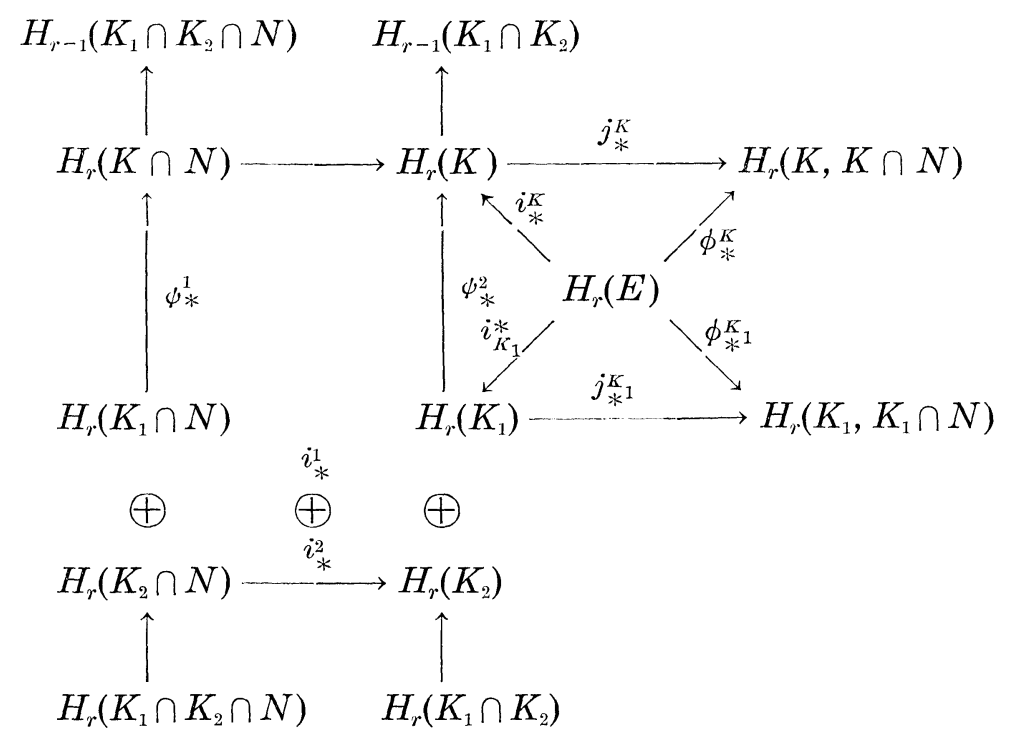

The two vertical sequences are Mayer-Vietoris sequences. Also, the two horizontal sequences are exact. We have

$$
H_{r-1}\left(K_{1} \cap K_{2}\right)=H_{r-1}\left(K_{1} \cap K_{2} \cap N\right)=H_{r}\left(K_{1} \cap K_{2}\right)=H_{r}\left(K_{1} \cap K_{2} \cap N\right)=0,
$$

since $K_{1} \cap K_{2}$ is a $T_{r-1}$ set. Since $\phi_{*}^{K}\left(Z_{r}\right)=j_{*}^{K} i_{*}^{K}\left(Z_{r}\right)=0$, there exists a $Z_{r}^{3} \in H_{r}(K \cap N)$ such that $i_{*}^{3}\left(Z_{r}^{3}\right)=i_{*}^{K}\left(Z_{r}\right)$. There exists

$$
\left(Z_{r}^{1}, Z_{r}^{2}\right) \in H_{r}\left(K_{1} \cap N\right) \oplus H_{r}\left(K_{2} \cap N\right)
$$

such that $\psi_{*}^{1}\left(Z_{r}^{1}, Z_{r}^{2}\right)=Z_{r}^{3}$. By commutativity,

$$
\psi_{*}^{2}\left(i_{*}^{1}\left(Z_{r}^{1}\right), i_{*}^{2}\left(Z_{r}\right)\right) i_{*}^{2} \psi_{*}^{1}\left(Z_{r}^{1}, Z_{r}^{2}\right)=i_{*}^{3}\left(Z_{r}^{3}\right)=i_{*}^{K}\left(Z_{r}\right),
$$

and

$$
\psi_{2}^{*}\left(i_{* 1}^{K}\left(Z_{r}\right), 0\right)=i_{*}^{K}\left(Z_{r}\right)
$$

By exactness, $\psi_{*}^{2}$ is an isomorphism, hence $i_{*}^{1}\left(Z_{r}^{1}\right)=i_{*}^{K}\left(Z_{r}\right)$. Therefore, $j_{*}^{K} i^{K_{1}}\left(Z_{r}\right)=j_{*}^{K} i_{\text {* }}^{1}\left(Z_{r}^{1}\right)=0$. But this is impossible, since $K$ is minimal. Thus, $K=E$ and $\phi^{*}$ is one-to-one. 
3. Cyclic elements in locally connected spaces. The zero-dimensional cyclic elements in a locally connected continuum have several useful properties. For example, if the continuum $M$ is locally connected, then the zero-dimensional cyclic elements of $M$ are also locally connected and these cyclic elements form a null sequence. Also, the simple 0 -links (definition below) are identical with the $E_{0}$ sets in an $l c^{0}$ space [6]. The examples below show that these properties do not generalize.

Definition 3.1. A non-degenerate subset $K$ of $M$ is called a simple $r$-link of $M$, if $K$ is maximal with respect to the following property: if $M=M_{1} \cup M_{2}, T_{r}$-separated, then $K \subset M_{1}$ (or $K \subset M_{2}$ ). In other words, $K$ is a maximal subset which can not be separated by a $T_{r}$ set that also separates $M$.

LEMmA 3.2. All simple r-links in $M$ are closed. If $K_{1}$ and $K_{2}$ are two distinct simple r-links in $M$, then $K_{1} \cap K_{2}$ is a $T_{r}$ set. If $L$ is a non-degenerate subset of $M$ that is not disconnected by any $T_{r}$ set which also disconnects $M$, then $L$ is contained in a simple r-link of $M$.

Proof. The proof is similar to those for the corresponding lemmas for cyclic elements.

EXAMPLE. We will construct an $l c^{r}$ space $M$ in which the collection of $E_{r}$ sets does not form a null sequence. This example will also show that, in an $l c^{r}$ space, the simple $r$-links need not be the same as the $E_{r}$ sets.

For each positive integer $n$, let $R_{n}$ be a solid, three dimensional rod of height one and diameter $1 / 2^{n}$. In Euclidean three-space, define $I$ by $I=\{(x, y, z) \mid x=0, y=0,0 \leqq z \leqq 1\}$. Imbed $R_{n}$ in three-space so that $R_{n}$ is tangent to $R_{n+1}$ and the sequence of sets $R_{n}$ converges to $I$ (i.e. $\left.R_{n}=\left\{(x, x, z) \mid x^{2}+\left(y-3 / 2^{n+1}\right)^{2} \leqq 1 / 2^{2 n+2}, 0 \leqq z \leqq 1\right\}\right)$. Let $M$ be the set $\left[\bigcup_{n=1}^{\infty} R_{n}\right] \cup I$. Then $M$ is a compact $1 c^{1}$ space, each $R_{n}$ is an $E_{1}$ set in $M$, but the collection $\left\{R_{n}\right\}$ is not a null sequence. Also, $I$ is a simple 1-link, but is not an $E_{1}$ set.

Theorem 3.3. If $M$ is $s-l c$ and $E$ is an $E_{r}$ set of $M$, where $s \geqq r$, then $E$ is $s-l c$.

Proof. Given any $x \in E$, and an open set $U^{0}$ of $E$ containing $x$, then there exists an open set $U$ of $M$ such that $U \cap E=U^{0}$. Since $M$ is $s-l c$, there exists an open set $V$, containing $x$, such that $\bar{V} \subset U$ and any compact $s$-cycle in $V$ bounds on a compact subset of $U$. Let $Z_{s}$ be a compact cycle on $V \cap E=V^{0}$. Then there exists a minimal 
closed set $K$ in $M$ such that $\bar{V}^{0} \subset K \subset U$, and $Z_{s}$ bounds on $K$. By using the Mayer-Vietoris sequence, as it was used in the proof of Theorem 2.4, we can show $K \subset U^{0}$. Therefore $Z_{s}$ bounds in $U^{0}$ and $E$ is $s-l c$.

EXAMPLE. We will construct a compact $l c^{r}$ space which contains an $E_{r}$ set which is not $l c^{r}$. Consider the following curve in three-space:

$$
x=0, y=t, z=\sin (\pi / t), \text { for } 0<t \leqq 1 .
$$

Expand this curve slightly so that it becomes a solid, three dimensional figure, which oscillates as it approaches the origin. Let $N$ be this space, along with its limiting line segment on the $z$-axis. Let $P=$ $\{(x, y, z) \mid x=0,0 \leqq y \leqq 1,-1 \leqq z \leqq 1\}$; then define $M=P \cup N$. Thus $N$ is an $E_{1}$ set in $M$ and $M$ is $l c^{1}$ but $N$ is not $0-l c$.

4. Cyclic elements and monotone mappings. A very basic property of the zero-dimensional cyclic element theory is the following: if $f: M \rightarrow$ $N$ is a monotone mapping (i.e. the inverse image of any point is connected), $M$ and $N$ are $l c^{0}$, and $E_{N}$ is an $E_{0}$ set in $N$; then there is an $E_{0}$ set in $M$ whose image under $f$ contains $E_{N}$. This result does not hold in higher dimensions, as the example below demonstrates. The best result we have obtained in this direction is Theorem 4.2.

Definition 4.1. A mapping $f: M \rightarrow N$ is $r$-monotone, if $H^{s}\left(f^{-1}(y)\right)=0$, for all $y \in N$ and $0 \leqq s \leqq r$.

THEOREM 4.2. Let $f$ be an $(r-1)$-monotone mapping of $M$ onto $N$, where $M$ and $N$ are compact Hausdorff spaces. If $D_{N}$ is a floor for $\gamma_{N}^{r} \in H^{r}(N)$, then there exists a floor $D_{M}$ for $f^{*}\left(\gamma_{N}^{r}\right)$ such that $f\left(D_{M}\right)=D_{N}$.

Proof. Since $f$ is $(r-1)$-monotone, $f^{*}: H^{r}(N) \rightarrow H^{r}(M)$ is a oneto-one mapping [1]. Therefore, $f^{*}\left(\gamma_{N}^{r}\right) \neq 0$. Consider the commutative diagram below. The vertical mappings are inclusion mappings; and $D_{M}$ is defined below.

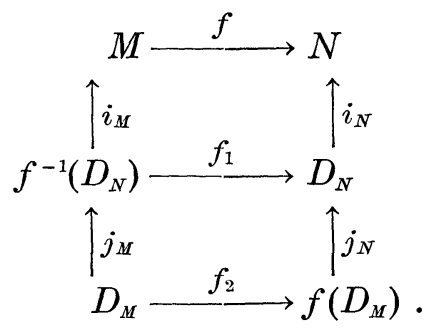

The mapping $f_{1}$ is the restriction of $f$ to $f^{-1}\left(D_{N}\right)$. Therefore, $f_{1}$ is $(r-1)$-monotone. Since $D_{N}$ is a floor for $\gamma_{N}^{r}, i_{N}^{*}\left(\gamma_{N}^{r}\right) \neq 0$. Since 
$f_{1}^{*}: H^{r}\left(D_{N}\right) \rightarrow H^{r}\left(f^{-1}(D)\right)$ is one-to-one, $i_{M}^{*} f^{*}\left(\gamma_{N}^{r}\right)=f_{1}^{*} i_{N}^{*}\left(\gamma_{N}^{r}\right) \neq 0$. Therefore, $f^{-1}\left(D_{N}\right)$ contains a floor for $f^{*}\left(\gamma_{N}^{r}\right)$. Denote this floor by $D_{M}$ and let $f_{2}$ be the restriction of $f$ to $D_{u}$. By the definition of a floor, $j_{M}^{*} i_{M}^{*} f^{*}\left(\gamma_{M}^{r}\right) \neq 0$. Since $j_{M}^{*} i_{M}^{*} f^{*}\left(\gamma_{N}^{r}\right)=f_{2}^{*} j_{N}^{*} i_{N}^{*}\left(\gamma_{N}^{r}\right)$, we have $j_{N}^{*} i_{N}^{*}\left(\gamma_{N}^{r}\right) \neq 0$. This implies $f\left(D_{M}\right)=D_{N}$, since $D_{N}$ is a floor for $\gamma_{N}^{r}$.

We shall omit the proofs of Lemmas 4.3 and 4.5.

Lemma 4.3. Let $N_{1}$ and $N_{2}$ be subsets of $M$ which can not be disconnected by a $T_{r}$ set. Suppose that $\bar{N}_{1} \cup \bar{N}_{2}$ is not a $T_{r}$ set. Then $\bar{N}_{1} \cup \bar{N}_{2}$ can not be disconnected by a $T_{r}$ set.

Lemma 4.4. Let $f: M \rightarrow N$, and suppose $T \subset N$ is a $T_{r}$ set such that $f^{-1}(T)$ is also a $T_{s}$ set. Also, assume $f$ is a homeomorphism of $M-f^{-1}(T)$ onto $N-T$. Then, if $T^{N}$ is a $T_{r}$ set in $N, f^{-1}\left(T^{N}\right)$ is a $T_{r}$ set in $M$.

Proof. Let $K$ be a closed subset of $f^{-1}\left(T^{N}\right)$. Denote $f^{-1}(T)$ by $T^{-1}$. In the commutative diagram below $f_{1}^{*}$ is an isomorphism, by excision. Therefore, by exactness, $H^{r}(K)=0$.

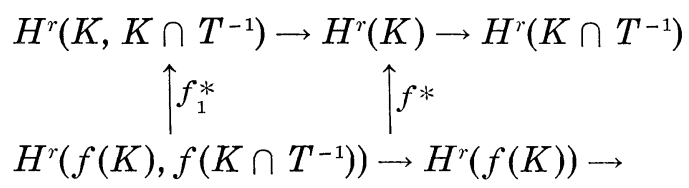

Lemma 4.5. Assume $f$ is a mapping of $M$ onto $N$ such that the inverse image of any $T_{r}$ set in $N$ is a $T_{r}$ set in $M$. If $K \subset M$ can not be disconnected by a $T_{r}$ set in $M$, then $f(K)$ can not be disconnected a $T_{r}$ set in $N$.

ExAMPLE. If $f$ is an $r$-monotone mapping of $M$ onto $N$, where $M$ and $N$ are $l c^{\infty}$ spaces and $E^{N}$ is an $E_{r}$ set in $N$; there may not be an $E_{r}$ set, $E^{M}$, in $M$ such that $f\left(E^{M}\right) \supset E^{N}$.

We will construct the example in three space. Consider the following solid cylinders:

$$
\begin{aligned}
& M_{1}=\left\{(x, y, z) \mid x^{2}+y^{2} \leqq 1,0 \leqq z \leqq 1\right\} \\
& M_{2}=\left\{(x, y, z) \mid x^{2}+(y-2)^{2} \leqq 1,0 \leqq z \leqq 1\right\}
\end{aligned}
$$

The cylinders $M_{1}$ and $M_{2}$ are tangent along $I=\{(x, y, z) \mid x=0$, $y=1,0 \leqq z \leqq 1\}$. Let $M_{3}$ be an arc joining the endpoints of $I$, which does not meet $M_{1} \cup M_{2}$ except at these endpoints. Let $M=\bigcup_{i=1}^{3} M_{i}$. We will define a decomposition of $M$, and will let $f: M \rightarrow N$ be the decomposition mapping. 
To form $N$, identify all the points in $M_{3}$ into a single point. Then the mapping $f: M \rightarrow N$ is $r$-monotone for all $r$ and the restriction of $f$ to $M-M_{3}$ is a homeomorphism.

We will show that $N$ is an $E_{1}$ set. First, neither $M_{1}$ nor $M_{2}$ can be disconnected by a $T_{1}$ set. Lemmas 4.4 and 4.5 imply that neither $f\left(M_{1}\right)$ nor $f\left(M_{2}\right)$ can be disconnected by a $T_{1}$ set. By Lemma $4.3, N=$ $f\left(M_{1}\right) \cup f\left(M_{2}\right)$ can not be disconnected by a $T_{1}$ set, since $f\left(M_{1}\right) \cup f\left(M_{2}\right)$ contains an essential 1-cycle. If $K$ is a closed subset of $M$ such that $f(K) \supset N$, then $K \supset M_{1} \cup M_{2}$. Then $K$ can be disconnected by a $T_{1}$ set, namely $M_{1} \cap M_{2}$. Therefore, there is no $E_{1}$ set in $M$ whose image is $N$.

Note that $M$ is obviously $l c^{r}$ for all $r$. Therefore $N$ is also $l c^{r}$, for all $r$, since $f$ is $r$-monotone, for all $r$.

\section{REFERENCES}

1. E. G. Begle, The Vietoris mapping theorem for bicompact spaces, Annals of Math.,

(2) 51 (1950), 534-542.

2. Samuel Eilenberg and Norman Steenrod, Foundations of Algebraic Topology, Princeton, 1952.

3. W. J. Puckett, Jr., A theorem concerning homologies in a compact space, Fundamentae Mathematicae, 31-32 (1938), 32.

4. Arthur Simon, N-Cyclic elements, Duke Math. Journal, 24 (1957), 1-7.

5. G. T. Whyburn, Cyclic elements of higher order, Amer. J. Math., 56 (1934), 133-146.

6. _ Analytic Topology, Amer. Math. Soc. Coll. Pub., 1942.

7. R. L. Wilder, Topology of Manifolds, Amer. Math. Soc. Coll. Pub. 1949.

CALIFORNIA INSTITUTE OF TEChNOLOGY 


\section{PACIFIC JOURNAL OF MATHEMATICS}

EDITORS

David Gilbarg

Stanford University

Stanford, California

R. A. Beaumont

University of Washington

Seattle 5 , Washington
A. L. Whiteman

University of Southern California

Los Angeles 7, California

L. J. PAIGE

University of California

Los Angeles 24, California

\section{ASSOCIATE EDITORS}

\author{
E. F. BECKENBACH \\ C. E. BURGESS \\ E. HEWITT \\ A. HORN
}

\author{
V. GANAPATHY IYER \\ R. D. JAMES \\ M. S. KNEBELMAN \\ L. NACHBIN
}
I. NIVEN
E. G. STRAUS
T. G. OSTROM
G. SZEKERES
H. L. ROYDEN
F. WOLF
M. M. SCHIFFER
K. YOSIDA

\section{SUPPORTING INSTITUTIONS}

\author{
UNIVERSITY OF BRITISH COLUMBIA \\ CALIFORNIA INSTITUTE OF TECHNOLOGY \\ UNIVERSITY OF CALIFORNIA \\ MONTANA STATE UNIVERSITY \\ UNIVERSITY OF NEVADA \\ OREGON STATE COLLEGE \\ UNIVERSITY OF OREGON \\ OSAKA UNIVERSITY \\ UNIVERSITY OF SOUTHERN CALIFORNIA
}

\author{
STANFORD UNIVERSITY \\ UNIVERSITY OF TOKYO \\ UNIVERSITY OF UTAH \\ WASHINGTON STATE COLLEGE \\ UNIVERSITY OF WASHINGTON \\ $* \quad * \quad *$ \\ AMERICAN MATHEMATICAL SOCIETY \\ CALIFORNIA RESEARCH CORPORATION \\ HUGHES AIRCRAFT COMPANY \\ SPACE TECHNOLOGY LABORATORIES
}

Mathematical papers intended for publication in the Pacific Journal of Mathematics should be typewritten (double spaced), and the author should keep a complete copy. Manuscripts may be sent to any one of the four editors. All other communications to the editors should be addressed to the managing editor, L. J. Paige at the University of California, Los Angeles 24, California.

50 reprints per author of each article are furnished free of charge; additional copies may be obtained at cost in multiples of 50 .

The Pacific Journal of Mathematics is published quarterly, in March, June, September, and December. The price per volume (4 numbers) is $\$ 12.00$; single issues, $\$ 3.50$. Back numbers are available. Special price to individual faculty members of supporting institutions and to individual members of the American Mathematical Society: $\$ 4.00$ per volume; single issues, $\$ 1.25$.

Subscriptions, orders for back numbers, and changes of address should be sent to Pacific Journal of Mathematics, 2120 Oxford Street, Berkeley 4, California.

Printed at Kokusai Bunken Insatsusha (International Academic Printing Co., Ltd.), No. 6, 2-chome, Fujimi-cho, Chiyoda-ku, Tokyo, Japan.

PUBLISHED BY PACIFIC JOURNAL OF MATHEMATICS, A NON-PROFIT CORPORATION

The Supporting Institutions listed above contribute to the cost of publication of this Journal, but they are not owners or publishers and have no responsibility for its content or policies. 


\section{Pacific Journal of Mathematics}

\section{Vol. 9, No. 4 \\ August, 1959}

Frank Herbert Brownell, III, A note on Kato's uniqueness criterion for

Schrödinger operator self-adjoint extensions ............... 953

Edmond Darrell Cashwell and C. J. Everett, The ring of number-theoretic

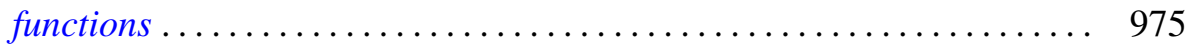

Heinz Otto Cordes, On continuation of boundary values for partial

differential operators ............................. 987

Philip C. Curtis, Jr., n-parameter families and best approximation . . . . . . 1013

Uri Fixman, Problems in spectral operators . . . . . . . . . . . . . . . 1029

I. S. Gál, Uniformizable spaces with a unique structure .............. 1053

John Mitchell Gary, Higher dimensional cyclic elements ............ 1061

Richard P. Gosselin, On Diophantine approximation and trigonometric

polynomials ..................................... 1071

Gilbert Helmberg, Generating sets of elements in compact groups ........ 1083

Daniel R. Hughes and John Griggs Thompson, The H-problem and the

structure of $H$-groups .................................. 1097

James Patrick Jans, Projective injective modules ................. 1103

Samuel Karlin and James L. McGregor, Coincidence properties of birth and

death processes ..................................... 1109

Samuel Karlin and James L. McGregor, Coincidence probabilities ........ 1141

J. L. Kelley, Measures on Boolean algebras ................... 1165

John G. Kemeny, Generalized random variables ................... 1179

Donald G. Malm, Concerning the cohomology ring of a sphere bundle ... . . 1191

Marvin David Marcus and Benjamin Nelson Moyls, Transformations on

tensor product spaces .................................. 1215

Charles Alan McCarthy, The nilpotent part of a spectral operator ........ 1223

Kotaro Oikawa, On a criterion for the weakness of an ideal boundary

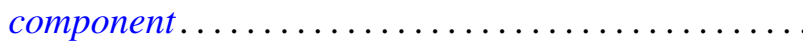

Barrett O'Neill, An algebraic criterion for immersion . . ............... 1239

Murray Harold Protter, Vibration of a nonhomogeneous membrane ... . . . . 1249

Victor Lenard Shapiro, Intrinsic operators in three-space . . . . . . . . . . . 1257

Morgan Ward, Tests for primality based on Sylvester's cyclotomic

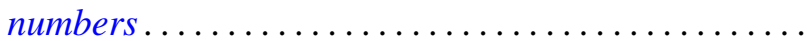

L. E. Ward, A fixed point theorem for chained spaces ....

Alfred B. Willcox, Šilov type $C$ algebras over a connected locally compact

abelian group..................................... 1279

Jacob Feldman, Correction to "Equivalence and perpendicularity of

Gaussian processes" ........................ 